\title{
A Tribute to Nigel J. Kalton (1946-2010)
}

\author{
Peter G. Casazza, Coordinating Editor
}

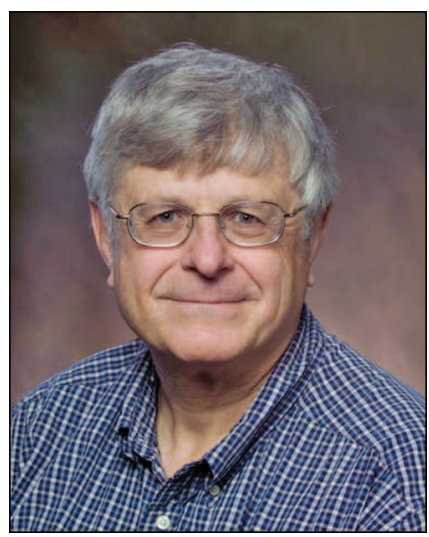

Nigel Kalton at his sixtieth birthday conference.
Nigel Kalton was born June 20, 1946, in Bromley, England. The name Kalton is an anglicized form of the original German family name, Kaltenbach, which is actually the name which appears on Nigel's birth certificate. He was the son of Gordon and Stella Kalton. Nigel was educated at Dulwich College, where he excelled at both mathematics and chess. He played chess on the college team with Ray Keene. Keene later became Britain's second chess grandmaster despite losing once to Kalton in the Dulwich College championship.

Nigel then studied mathematics at Trinity College, Cambridge, where he met his wife, Jennifer, to whom he was married for forty-one years. They later had two children, Neil and Helen, and four grandchildren. He and Keene played chess for Trinity College. He twice came in second in the Cambridge University chess championship. He also represented Cambridge in the varsity match against Oxford. For the rest of

Peter Casazza is a curators' professor at the University of Missouri. His email address is casazzap@mi ssouri . edu. DOI: http://dx.doi.org/10.1090/noti868 his career, Kalton was a very competitive chess player, winning the Major Open at the British Championship in 1970. Some of his games can still be found on the Internet (follow the link: http://ka7tonmemorial.missouri.edu/chess).

Kalton continued his graduate studies in mathematics at Cambridge University under Dr. D. J. H. (Ben) Garling, working in Banach space theory. He received the Ph.D. in 1970. His thesis was awarded the Rayleigh Prize for research excellence. He then held positions at Lehigh University in Pennsylvania, Warwick, and Swansea before moving permanently to the United States. This move was prompted by a variety of factors such as salary, working conditions, research grants, and access to other specialists in his area. He taught at the University of Illinois and Michigan State University and then in 1979 moved to the University of Missouri, where he spent the rest of his career.

Kalton received a number of honors and awards, including the prestigious Stefan Banach Medal, named after the founder of Nigel's chosen field, Banach space theory. This prize was established by the Polish Academy of Sciences in 1992 in honor of the centenary of Banach's birth.

Nigel Kalton was at the very top of his field in productivity, creativity, depth and breadth of knowledge, as well as research support, invited addresses, citations, and the view of his peers. There are a number of fundamental results in mathematics that carry his name, including Kalton spaces, Kalton operators, and much more. Nigel was very broad in his research, proving fundamental results in a dozen different areas of research. He had more than two hundred seventy research articles (which 
averaged over twenty-five pages each) and books and was on the editorial board of six mathematics journals. Nigel believed that every math paper must have at least one significant theorem that justified its existence, and he lived by this his whole career.

Nigel was famously competitive at anything he attempted, including mathematics, chess, racquetball, etc. He spent a lot of time with his children and grandchildren, but they complained that he never let them win at anything. One time when we were playing racquetball, my head was split open by a racquet and I hit the floor squirting blood everywhere. Nigel stepped over me and won the shot, then turned around to ask if I was all right. I said, "Nigel, what the hell was that?" He replied, "Pete, I don't trust you not to split your head open just to win the point."

Kalton was a very powerful mathematician who could learn new mathematics at an incredible rate. People from our department would come into his office and ask for help with their research, even in areas in which Nigel had never worked. He would tell them to put the definitions and the problem on the board. Invariably, in a few days he would have a solution for them. One time Jerry Lange of our department came to Nigel with a problem in continued fractions he had been working on for fifteen years. Nigel solved it in forty-eight hours, and later this solution was used to answer a problem of Ramanujan. Another time, Gilles Pisier gave a talk on an approach by Le Merdy to an open problem due to the Russian mathematician Vladimir Peller. Kalton was in the audience and quickly resolved the problem in a joint publication with Le Merdy in 2002.

Kalton was setting up a research group at the University of Missouri and hired me into the group in 1983. For the first two years we talked mathematics but could not find any common interests. Then I spent a year at the Hebrew University of Jerusalem. Nigel came to visit for a month during that year, and we wrote our first paper together. I quickly learned that you do not start a research project with Nigel or you would spend all your time playing catch-up trying to understand today what Nigel had done yesterday. Although I wrote a number of papers with him, they often occurred because I worked on something for months before showing it to him. Even then, within a short time I was again playing catch-up. Kalton had an amazingly universal view of mathematics. One time someone in our department asked him if a certain inequality was true in a particular Banach space. Within twenty-four hours Nigel had classified all Banach spaces in which this inequality was true-and the space in question was just one of these. We used to call Kalton the bulldozer, since given just one new idea, he could plow his way through an entire field in a matter of weeks to solve a famous problem.

Having an office next to Nigel for twentyseven years could have been the most humbling experience of my life. But because of who he was, it wasn't. He was a towering figure in mathematics but maintained a humble attitude towards others. He was always ready to help anyone who wanted to learn mathematics. He was a man of complete honesty in an area which was not always known for its honesty. He was a man of integrity in an area not always known for its integrity. He was a man of courage who spoke up against injustice when others were afraid to speak up.

I always thought of Nigel as someone who had too big a brain

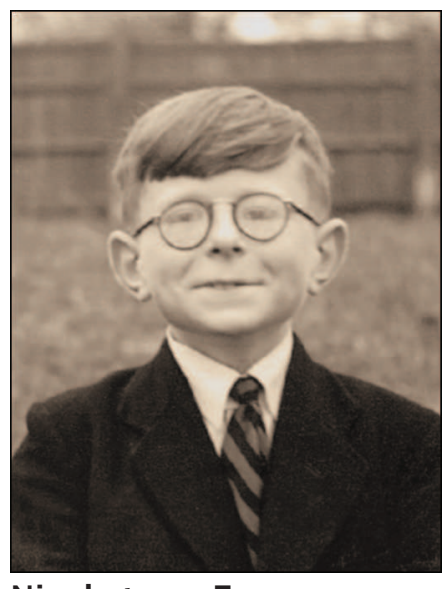

Nigel at age 7. and just could not find enough to keep it challenged. Every day he scanned the Internet for all the news of the world and then scanned all new journals in the library looking for new ideas and new problems. One day I was at the board in his office while we were working out a problem. Every ten minutes or so he would go to his computer, type in a few things, and then come back to the discussion. When I asked what was happening, he said he was playing chess with someone in England. I am sure his opponent had no idea that Nigel was not concentrating on the game full time but instead was making instant decisions in a matter of seconds in between proving a theorem. It was certainly fun and exciting to be able to go next door every day and talk mathematics with a giant. But it was just as much fun to talk to someone who was a superior intellect on all subjects. Also, Nigel prided himself on being a history buff who knew the history of most areas of the world.

Kalton was instrumental in building the mathematics department at the University of Missouri into an internationally recognized department. He consistently provided leadership within the department and across the campus. He was a standout amongst the over 1,500 faculty at the University of Missouri. He was appointed the Luther Marion Defoe Distinguished Professor of Mathematics (1984) and he was appointed to the Houchins Chair of Mathematics (1985). He received the Chancellors Award for Outstanding Research (1984), and the Weldon Springs Presidential Award for outstanding research (1987). In 1995 he received the highest recognition bestowed by the University of Missouri System by being appointed a curators' professor. 


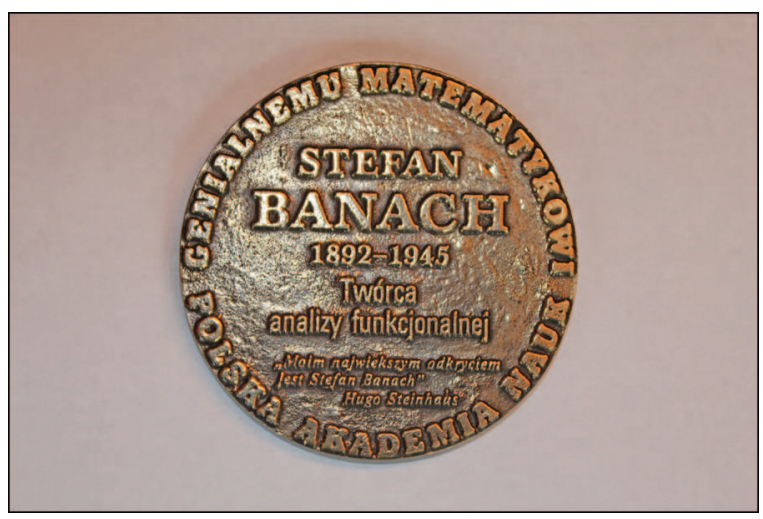

Nigel's Banach medal.

As mathematicians we spend our whole lives living a public lie. It is not by choice; it comes with the territory. We put forward an image of absolute confidence even when we doubt our own abilities. We act like we are invincible when we know we are human. We delude ourselves into thinking that mathematics is the most important thing in the universe because we couldn't live with devoting our entire being to something which is not the most important thing. We wonder if anyone really likes us or if they just respect us, and if so, will they no longer like us as we get older and our abilities start to fade? Contrary to popular belief, Nigel was human, but he knew all of the above. He did not live in a dream world. He knew we should all be doing this just because it is fun and challenging, and if he was going to do it, then his competitive streak forced him to be the best at it.

On August 31, 2010, the mathematical world lost a giant of a human being and a giant of a mathematician. I lost my best friend. We are all much the less for it.

Fritz Gesztesy of the University of Missouri mathematics department has set up a spectacular website to honor Kalton's memory and achievements. It contains (with the publishers' permission) almost all of his publications, and there people can post memories of Nigel (http: //kal tonmemorial . mi ssouri . edu).

\section{Aleksander Pełczyński}

\section{Nigel Kalton and Universal Spaces}

The first international meeting attended by Nigel Kalton was the Conference on Operator Ideals and Nuclear Space (Warsaw, Poland, in August 1969). He came with his thesis advisor, Professor Ben Garling. He delivered a 30-minute talk "Bases in

Aleksander Petczyński is professor of mathematics at the Polish Academy of Sciences, Warsaw, Poland. His email address is o1ek@impan.gov.p1. locally convex spaces". Then we met for the first time. I got the opportunity to know Nigel better and to recognize his unusual mathematical personality in the summer of 1972 while visiting for a couple of weeks at the University College of Swansea. I witnessed when he began research on universal spaces and universal bases in metric linear spaces which eventually resulted in the remarkable paper [K1] submitted to Studia Math. in 1975. In this essay I would like to describe some results from this early paper by Nigel Kalton, which was the starting point of his important investigation on the structure of general metric linear spaces.

In the early seventies the vast majority of research in linear functional analysis was devoted to Banach spaces, although considerable attention was still paid to locally convex linear topological spaces, in particular to nuclear spaces. There were very few papers dealing with nonlocally convex metric linear spaces, usually providing counterexamples to various conjectures. The paper [K1] was different. It showed that the structure theory of F-spaces (= complete metric linear spaces) is rich, complicated, and interesting. In particular, Kalton constructed new objects having various universal properties for several classes of F-spaces. He generalized many results on Banach spaces and Frechet spaces (= complete metric linear locally convex spaces), at the same time improving the results in the original setting.

Solving a problem of S. Rolewicz [R, Problem II.4.1], Nigel constructed a separable F-space isomorphically universal for all separable metric linear spaces, i.e., a space $U F$ such that every separable metric linear space is isomorphic (= linearly homeomorphic) to a subspace of $U F$. The result completes the classical fact discovered by Banach and Mazur [B, p. 185] that the space $C[0,1]$ of all scalar-valued continuous functions on the unit interval is isometrically universal for all separable normed linear spaces and the observation of Mazur and Orlicz [MO] that the Frechet space of all continuous scalar-valued functions on the real line is isomorphically universal for all separable Frechet spaces. Kalton's construction of the space $U F$ is based upon an essential improvement of the "packing technique" originated in the setting of Banach spaces by V. I. Gurarii [G] and used by the author [P1] and M. I. Kadec [Ka].

There is a co-universal object for all separable Fspaces (cf. [K1, Theorem 5.4]): There is a separable $F$-space CoF such that every separable F-space is a quotient of CoF. The proof uses a result of Turpin $[\mathrm{T}]$ that every F-space is a quotient of an F-space with an absolute basis.

Recall that a basis in F-space $X$ is a sequence $\left(e_{n}\right)$ of elements of $X$ such that for every $x \in X$ there is a unique sequence of scalars $\left(t_{n}\right)$ such 
that $x=\sum_{n} t_{n} x_{n}$. A basis is unconditional if the series $\sum_{n} t_{n} x_{n}$ converges unconditionally for every $x \in X$. A basis $\left(e_{n}\right)$ is absolute if the topology of $X$ is determined by an equivalent F-norm, say $q($.$) such that q(x)=\sum_{n} q\left(t_{n} x_{n}\right)$. An F-norm is a nonnegative function $q($.$) on X$ which satisfies the triangle inequality, is translation invariant, $q(x)>0$ for $x \neq 0, q(t x) \leq q(x)$ for scalars $t$ with $|t| \leq 1, \lim _{t=0} q(t x)=0$ for $x \in X$. For separable Banach spaces the co-universal object is the space $\ell_{1}$ of all absolutely convergent scalar sequences. This observation goes back to J. Schauder.

The existence of isomorphically universal objects for some special classes of F-spaces is also discussed. A big portion of [K1] is devoted to the study of complementably universal spaces and complementably universal bases.

Recall that a linear topological space $X$ is complementably universal for the family $\mathcal{F}$ of linear topological spaces if every member of $\mathcal{F}$ is isomorphic to a subspace of $X$ which is complemented in $X$, i.e., is a range of a linear projection of $X$ into itself. A basis $\left(e_{n}\right)$ in a linear topological space $E$ is complementably universal for a family $\mathcal{B}$ of bases in linear topological spaces if for every member of $\mathcal{B}$, say $\left(b_{k}\right)$, there is a subsequence $\left(n_{k}\right)$ such that the map $b_{k} \rightarrow e_{n_{k}}$ for $k=1,2, \ldots$ extends to an isomorphic embedding of the space spanned by $\left(b_{k}\right)$ into $E$. Moreover, there is a linear projection of $E$ into a subspace spanned by $\left(e_{n_{k}}\right)$ which takes $e_{n}$ to 0 for $n \neq n_{k}(k=1,2, \ldots)$.

In [P1] the isomorphically unique separable Banach space $U B$ was constructed with a complementably universal basis for the family of all bases $\left(b_{k}\right)$ in all separable Banach spaces satisfying the condition $0<\inf _{k}\left\|b_{k}\right\| \leq \sup _{k}\left\|b_{k}\right\|<\infty$. The result has been improved in [K1, Theorem 3.2], where a complementably universal basis was built without any normalization condition. The argument in [K1] is considerably simpler than that in [P1]. It uses the Banach-Mazur isometric universality of $C[0,1]$ and a clever renorming of a linearly independent sequence in a normed space transforming the sequence into a basis in the new normed space. Essentially the same argument was invented by G. Schechtman [Sc]. Unfortunately, because of lack of information in the book [LT], the paper [K1] is not mentioned, only [Sc]. The renorming technique for universal bases goes back to Zippin [Z]. Theorem 3.2 is extended on Frechet spaces [K1, Theorems 3.5-3.6]: There is an isomorphically unique Frechet space with complementably universal basis for the family of all bases in Frechet spaces. A similar result holds for the family of all Frechet spaces with unconditional bases.

Recall that a separable F-space has BAP (= bounded approximation property) if the identity operator on the space is a pointwise limit in the topology of the space of a sequence of linear operators of finite rank. In contrast with Frechet spaces, the existence of an F-space without $B A P$ is easy because there are F-spaces without nontrivial linear functionals. Having $B A P$ is a weaker property than having a basis. If a Frechet space (resp., Banach) has $B A P$, then it is isomorphic to a complemented subspace of a Frechet space (resp., Banach) with a basis [P2], [JRZ]. Thus a complementably universal space for all separable Frechet (resp.,

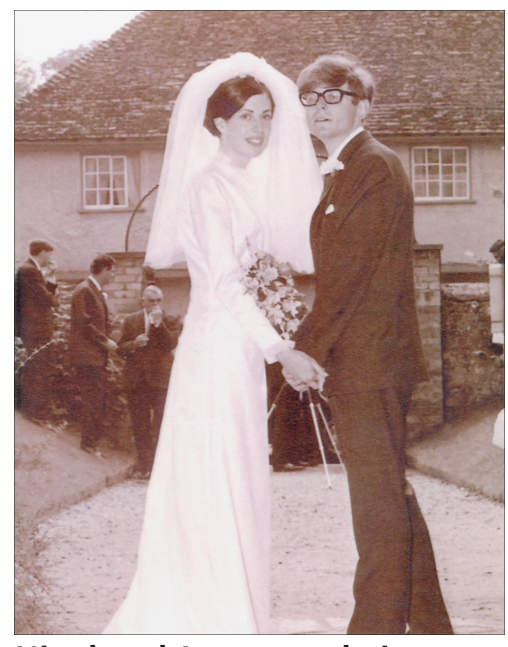

Nigel and Jenny on their wedding day, 1969. Banach) spaces with bases is complementably universal for all Frechet (resp., Banach) spaces with $B A P$. The separable Banach space complementably universal for all separable Banach spaces with $B A P$ was first constructed by Kadec [Ka]. Johnson and Szankowski [JS] proved that there is no separable Banach space complementably universal for all separable Banach spaces.

The problem whether there is an F-space with a basis which is complementably universal for the family of all F-spaces with bases remains open. In an attempt to solve this problem, Nigel Kalton was able to prove [K1, Theorem 4.4]: There is a unique F-space with a basis and without arbitrary short lines which is complementably universal for the family of all F-spaces with bases and without arbitrary short lines.

Recall that an F-space has arbitrary short lines if in every neighborhood of zero of the space there is the line (= one-dimensional subspace). In contrast with Frechet spaces it is not true that every separable F-space with $B A P$ is isomorphic to a complemented subspace of an F-space with a basis. At the end of [K1] there is an ingenious counterexample (two and a half pages)! However, it is true that every F-space with $B A P$ is isomorphic to a complemented subspace of an F-space with a finite-dimensional Schauder decomposition; i.e., there is a sequence $\left(E_{n}\right)$ of finite-dimensional subspaces of the space such that every element $x$ of the space has the unique expansion $x=\sum_{n} e_{n}$ with $e_{n} \in E_{n}(n=1,2, \ldots)$.

In [K1] other universal space problems for quasi-Banach spaces are studied. Recall that a quasi-Banach space is a $p$-Banach space, i.e., a complete $p$-normed space. A $p$-normed space is a metric linear space $X$ whose topology is 


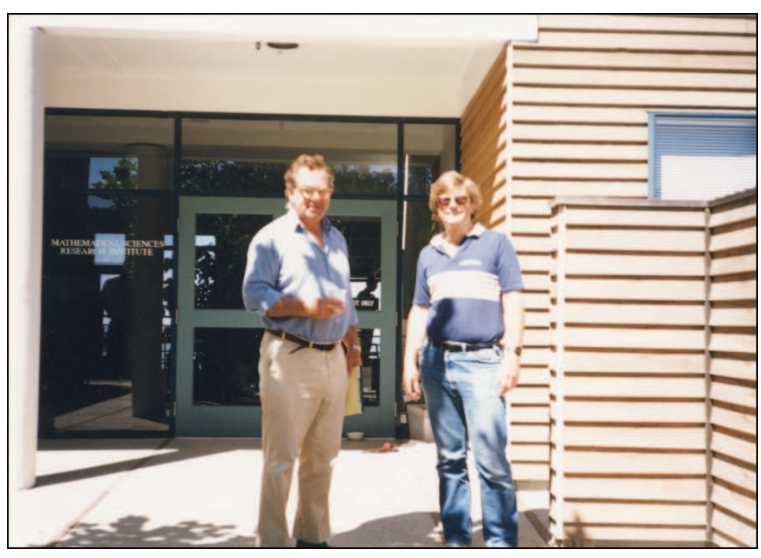

Nigel and his Ph.D. advisor, Ben Garling, at MSRI.

determined by an F-norm $q($.$) satisfying the$ condition

$$
q(t x)=|t|^{p} q(x) \quad(x \in X ; \quad t \text { scalars }) .
$$

The following facts are established in [K1]: Let $p$ with $0<p \leq 1$ be fixed. Then

(i) There exists a separable $p$-Banach space isomorphically universal for the family of all separable $p$-normed spaces.

(ii) There exists an isomorphically unique $p$ Banach space $B_{p}$ with a complementably universal basis for the family of all bases in $p$-Banach spaces.

(iii) Every $p$-Banach space with $B A P$ is isomorphic to a complemented subspace of a $p$ Banach space with a basis. In particular, $B_{p}$ is complementably universal for the family of all $p$-Banach spaces with bases.

Note that if $0<p<1$, then the Lebesgue space $L_{p}(0,1)$ is a $p$-Banach space without $B A P$ because it does not have nontrivial linear functionals. Let us mention some nonexistence results on universal $p$-Banach spaces that use the technique of Szlenk type index [Sz], [K1, Theorem 6.8, Corollary 6.8]: For $0<p<1$ there is no separable $p$-normed F-space with separating dual which is isomorphically universal for all separable $p$-Banach spaces with separating duals.

There is no separable F-space which is complementably universal for all $p$-Banach spaces with separating duals.

The results of [K1] are only a sample of Kalton's contribution to the theory of metric linear spaces (cf. [KPR]), which constitutes a small part of his contribution to modern functional analysis. He created the theory of quasi-Banach spaces and showed that it is a natural completion of the theory of Banach spaces. He also exhibited in the theory of quasi-Banach spaces and beyond it the role of quasi-linear maps. He discovered, together with his collaborators, several applications of
quasi-Banach spaces and quasi-linear maps. In particular, he invented new techniques of constructing Banach spaces and exhibited important connections with interpolation theory (cf. the excellent surveys [K2], [KM]).

\section{References}

[B] S. BANACH, Théorie des Opérations Linéaires, Monografie Matematyczne 1, Warszawa, 1932.

[G] V. I. GURARIǏ, Spaces of universal rearrangement, isotropic spaces and Mazur's problem on the rotation of Banach spaces, Sibir J. Math. 7 (1966), 102-113 (in Russian).

[JS] W. B. JoHnson and A. SzANKowsKi, Complementably universal Banach spaces, Studia Math. 58 (1976), 9197.

[JRZ] W. B. JOHNSON, H. Rosenthal, and M. ZIPPIN, On bases, finite dimensional decompositions and weaker structures of Banach spaces, Israel J. Math. 9 (1971), 488-506.

[K1] N. J. KALTON, Universal spaces and universal bases in metric linear spaces, Studia Math. 61 (1977), 161-191.

[K2] _ Quasi-Banach spaces, Handbook of Geometry of Banach Spaces, W. B. Johnson and J. Lindenstrauss, editors, vol. II (2003), 1099-1130.

[KM] N. J. KALTON and S. MONTGOMERY-SMITH, Interpolation of Banach spaces, Handbook of the Geometry of Banach Spaces, W. B. Johnson and J. Lindenstrauss, editors, vol. II (2003), 1131-1175.

[KPR] N. J. Kalton, N. T. PeCK, and J. W. Roberts, An FSpace Sampler, London Math. Soc. Lecture Notes 89, Cambridge University Press, Cambridge, 1985.

[Ka] M. I. KADEC, On complementably universal Banach spaces, Studia Math. 40 (1971), 85-89.

[LT] J. LINDENSTRAUSS and L. TZAFRIRI, Classical Banach Spaces, Vol. I, Ergebn. Math. Grenzgeb. 92, Springer, 1977.

[MO] S. MAZUR and W. ORLICZ, Sur les espaces métriques linéaires (II), Studia Math. 13 (1953), 137-179.

[P1] A. PeŁcZYŃSKI, Universal bases, Studia Math. 32 (1969), 247-268.

[P2] _ Any separable Banach space with the bounded approximation property is a complemented subspace of a Banach space with a basis, Studia Math. 40 (1971), 239-243.

[R] S. Rolewicz, Metric Linear Spaces, Monografie Matematyczne 56, Warszawa, 1972.

[Sc] G. Schechtman, On Pełczyński's paper "Universal bases", Israel J. Math. 22 (1975), 181-184.

[Sz] W. SzLENK, The nonexistence of a separable reflexive Banach space universal for all separable reflexive Banach spaces, Studia Math. 30 (1968), 53-61.

[T] P. TURPIN, Convexités dans les espaces vectoriels topologiques généraux, Thése Orsay 1974, Diss. Math. 131 (1976).

[Z] M. ZIPPIN, Existence of universal members in certain families of bases in Banach spaces, Proc. Amer. Math. Soc. 26 (1970), 294-301. 


\section{Joe Diestel}

\section{Memories of Nigel Kalton}

I first met Nigel in the late 1970s. I had corresponded with him earlier and had read a number of his papers; in fact, my friend Steve Saxon was the first to alert me to some of what Nigel was doing. Steve told me that "this English guy was proving many of the James' theorems in general linear topological spaces in ways easier than the Banach space case!" I checked and discovered that this was so. Later Nigel wrote me about some observations I'd made regarding vector measures which he had also made, again with proofs far more elegant than mine and more general too. So I was very happy when I finally met the man in person.

I was in for the first of many surprises Nigel was to spring on me-a grand surprise, to be sure: his humble sense of humor. Once Nigel was describing his first talks at the Northern British Functional Analysis Seminar in Newcastle-UponTyne. He told how confident he felt, saying he was talking about life in nonlocally convex spaces, a topic few others were studying and almost certainly none that were at the seminar. "Imagine my surprise," said Nigel, "when at the end of my talk John Ringrose, the moderator that day, asked for comments or questions and from the back of the lecture hall came "I can prove your main theorem in a couple of lines!", and that was the day I first met Olek Pełczyński."

These stories emphasize a characteristic of Nigel Kalton that was most endearing: a subtle modesty cloaked in a fine sense of humor. He said on several occasions that he felt very lucky to make a living doing mathematics and usually with a wry smile would add, "I don't think I could have made it in anything else!". If you talked to him for a while, you realized he chose mathematics, not the other way round.

One more story exhibiting Nigel's sense of humor, even at his own expense. I asked him about a curious feature of his CV: namely, he wrote several papers in differential games, a subject far from linear topological spaces. Here's how Nigel explained it. He went to Northwestern on sabbatical to study and work with Alexandra Ionescu-Tulcea without checking. As luck would have it, Professor Tulcea was away the term Nigel came. He said it must be a case of mathematical genetics! His advisor at Cambridge, D. J. H. "Ben” Garling, had taken sabbatical at Lehigh University to work with Bill Ruckle, without checking, and sure enough, Ruckle had moved to Clemson! In

Joe Diestel is emeritus professor of mathematics at Kent State University. His email address is jdi este1@kent. edu. any case, Nigel's plans had to be adapted to the situation, so he sat around the lounge talking to various mathematicians and came to the belief that his most enjoyable mathematical conversations were with Professors Elliot and Friedman; hence he worked with them for a term. The end result: a Memoir of the AMS and a half dozen papers. Not bad work for a novice.

Through the years I've been asked to write letters attesting to Nigel, letters in support of Nigel receiving

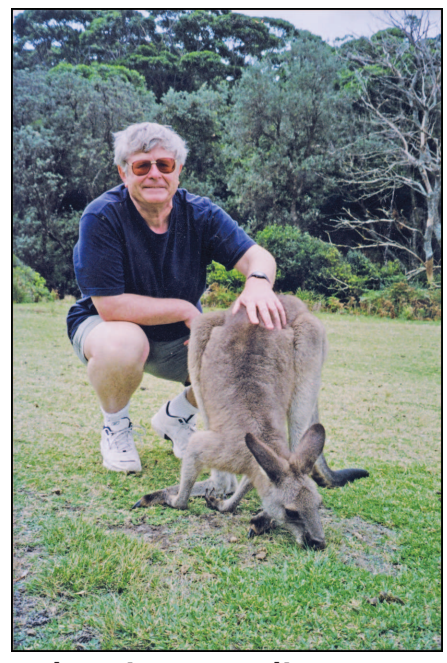

Kalton in Australia. some award or promotion. At first these were quite easy to write: review his papers, reflect on their standing with regard to the abstract analysis of an appropriate time period, and then summarize them in a grammatically correct manner. Of course, these letters could not avoid the distinctive character of the work and so were invariably very positive testimonials. As the years passed, it became harder to write an apt appraisal: Nigel's interests were ever more catholic in their scope and continued to broach new topics. His papers grew in frequency, length, and depth.

By the beginning of the new millennium he had started working in problems where delicate analysis of semigroups of operators held sway. I recall asking Nigel how he got interested in these problems; with characteristic humility he said it was an accident. He'd been visiting Gilles Lancien, who had recently become interested in the subject and had proven several results related to a problem of maximal regularity. Since Gilles was his host, he thought it proper that he be able to converse with Gilles on the problems that Gilles was thinking about. He took several papers back to his room to look at and over a very short period of time had developed his own Kalton insights. Again, his quickstudy ability soon led him to real understanding, and he was involved in proving some very special results. Of course, a natural consequence of Nigel's interest in these affairs led to yet another area in which he became a major contributor.

I had the pleasure of spending the AY 20002001 at the University of Missouri, Columbia, and as a result I saw Nigel in action and witnessed his interaction with colleagues and students. Many of Mizzou's most established mathematicians had worked with Nigel and were only too proud to mention how he'd contributed to their understanding of their own subject. Nigel was the cornerstone of that department's rapid rise. He 


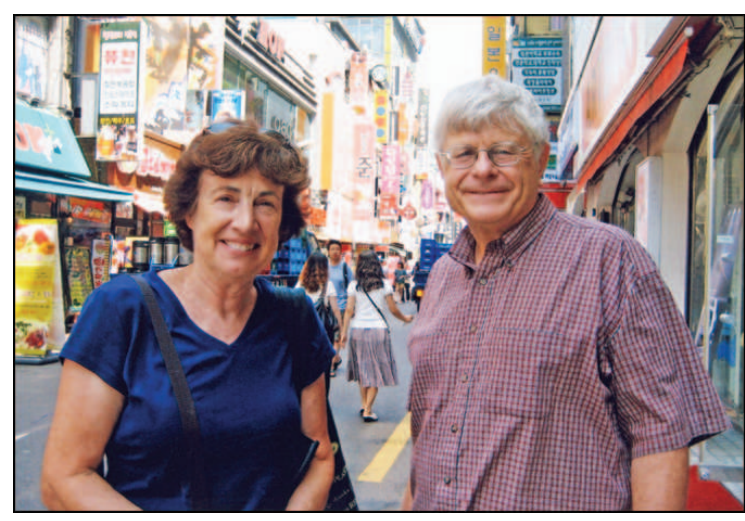

Nigel and Jenny in Korea, 2009.

was not just a colleague to many, but a friend as well.

\section{Roman Vershynin}

\section{Kalton and Quasi-Banach Spaces}

Nigel Kalton's general solution to the three-space problem [3], [4] was the first piece of serious research mathematics I got to read as an undergraduate student in 1995. The elegance of the construction of the Kalton-Peck space $Z_{2}$ and the technical power of those influential papers struck me then. Nigel became my first mathematical hero, and four years later, my Ph.D. advisor in Columbia, Missouri. Ironically, by that time our interests had somewhat diverged, as I had become more interested in geometric functional analysis. Although as a result we have not really collaborated, Nigel has remained to me what he had been in the first place, a master. Elegance and insight are the best words to describe his mathematics.

Among many areas of analysis Nigel worked in, the geometry of quasi-Banach spaces occupied a lot of his attention [6]. What distinguishes quasiBanach spaces from the more classical Banach spaces is the triangle inequality. In quasi-Banach spaces, the triangle inequality is allowed to hold approximately:

$$
\|x+y\| \leq C(\|x\|+\|y\|)
$$

for some constant $C \geq 1$. This relaxation leads to a broad class of spaces. Lebesgue spaces $L_{p}$ and Hardy spaces $H_{p}$ are Banach spaces for $1<p \leq \infty$ and only quasi-Banach spaces for $0<p<1$. In fact the topology of every locally bounded topological vector space $X$ can be induced by a suitable quasinorm.

Roman Vershynin is professor of mathematics at the University of Michigan. His email address is romanv@umich.edu.
Because of the weakness of the triangle inequality, convexity arguments do not work well in quasiBanach spaces (although the Aoki-Rolewicz theorem offers a remarkable surrogate, an equivalent quasi-norm $|\cdot|$ on $X$ which satisfies $|x+y|^{p} \leq$ $|x|^{p}+|y|^{p}$ for some $\left.0<p<1\right)$. Anyway, the absence of genuine convexity is a stumbling block that can make simple-looking problems difficult. For example, the atomic space problem, which asks whether every quasi-Banach space has a proper closed infinite-dimensional subspace, is still open. The most basic result of Banach space theory, the Hahn-Banach extension theorem, was shown by Kalton to fail in all quasi-Banach spaces that are not Banach spaces [2].

Kalton discovered surprising ways to put quasi-Banach spaces into action for problems about usual Banach spaces. One of the central questions in the field, the three-space problem, is about short exact sequences of Banach spaces

$$
0 \rightarrow X \rightarrow Z \rightarrow Y \rightarrow 0 .
$$

Such a space $Z$ is called a twisted sum of the spaces $X$ and $Y$. The three-space problem asks: what properties of $X$ and $Y$ are inherited by their twisted sums $Z$ [5]? For example, must $Z$ be isomorphic to a Hilbert space whenever both $X$ and $Y$ are?

The latter question was answered negatively by Enflo, Lindenstrauss, and Pisier in 1975 [1]. Shortly after that, Kalton and Peck developed an elegant approach to this problem and to twisted sums in general based on quasi-Banach spaces [4]. A remarkable product of this theory is the Kalton-Peck space $Z_{p}$ that is not isomorphic to $\ell_{p}$ while being a twisted sum of $X=Y=\ell_{p}$, where $1<p<\infty$.

The hidden link between twisted sums and quasi-Banach spaces is the notion of quasi-linear map, which is a homogeneous transformation $F: Y \rightarrow X$ that satisfies

$$
\left\|F\left(y_{1}+y_{2}\right)-F\left(y_{1}\right)-F\left(y_{2}\right)\right\| \leq K\left(\left\|y_{1}\right\|+\left\|y_{2}\right\|\right)
$$

with a suitable constant $K$. Quasi-linear maps can be easily constructed from twisted sums: the difference between a bounded homogeneous and a linear selection of the quotient map $Z \rightarrow Y$ is a quasi-linear map. Conversely, twisted sums can be constructed from quasi-linear maps: one can take the Cartesian product $Z:=X \times Y$ with the quasi-norm

$$
\|(x, y)\|:=\|x-F(y)\|+\|y\| .
$$

A trivial choice of $F=0$ and, more generally, of a linear map $F$ produces a twisted sum $Z$ that "splits" and is equivalent to the usual direct sum $X \oplus Y$. Any sufficiently nonlinear but quasi-linear map $F$ produces a nontrivial twisted sum, the one that does not split. In particular, going back to 
the original three-space problem for the Hilbert spaces $X=Y=\ell_{2}$, Kalton and Peck choose

$$
F\left(\sum_{i} x_{i} e_{i}\right)=\sum_{i}\left(\log \|x\|-\log \left|x_{i}\right|\right) x_{i} e_{i} .
$$

This produces the Kalton-Peck space $Z=Z_{2}$, a twisted sum of Hilbert spaces which itself is not isomorphic to a Hilbert space.

Of course, such constructions based on a quasilinear map $F$ can only get us a quasi-Banach space $Z$, even when $X$ and $Y$ are Banach spaces. On the one hand, this means that perhaps quasi-Banach rather than Banach spaces are the most natural habitat for twisted sums. On the other hand, an inspection of an earlier construction of Enflo, Lindenstrauss, and Pisier [1] led Kalton [3] to a crucial realization that a posteriori $Z$ is often (but not always) isomorphic to a Banach space. Fortunately, this always happens when $X$ and $Y$ are Hilbert spaces and more generally if $X$ and $Y$ are Banach spaces of nontrivial type (such as $\ell_{p}$ spaces with $1<p<\infty)$. So, most twisted sums automatically stay within the category of Banach spaces, even though their canonical construction goes deeply through quasi-Banach spaces!

Following its original discovery, Kalton-Peck's space $Z_{2}$ has become one of the main testing grounds for results and conjectures in Banach space theory. Its story continues up to the present day.

\section{References}

[1] P. Enflo, J. Lindenstrauss, and G. Pisier, On the "three space problem", Math. Scand. 36 (1975), no. 2, 199-210.

[2] N. J. Kalton, Basic sequences in F-spaces and their applications, Proc. Edinburgh Math. Soc. (2) 19 (1974/75), no. 2, 151-167.

[3] _ , The three space problem for locally bounded F-spaces, Compositio Math. 37 (1978), no. 3, 243-276.

[4] N. J. Kalton and N. T. PecK, Twisted sums of sequence spaces and the three space problem, Trans. Amer. Math. Soc. 255 (1979), 1-30.

[5] J. M. F. CASTILlo and M. GonzÁlez, Three-space problems in Banach space theory, Lecture Notes in Mathematics, 1667, Springer-Verlag, Berlin, 1997.

[6] N. J. KALton, Quasi-Banach spaces. Handbook of the Geometry of Banach Spaces, Vol. 2, pp. 1099-1130, North-Holland, Amsterdam, 2003.

\section{Gilles Godefroy}

\section{Nigel Kalton}

I met Nigel Kalton for the first time thirty years ago during my first Oberwolfach meeting in the summer of 1981. He delivered a lecture on symplectic Banach spaces with no Lagrangian subspaces (a joint work with R. C. Swanson), and although his exposition was perfect, I have to say that most of that lecture was well above my head. As ignorant as I could be at the time, I still knew that Nigel was a world expert on nonlocally convex spaces. Hence three years later, I made the

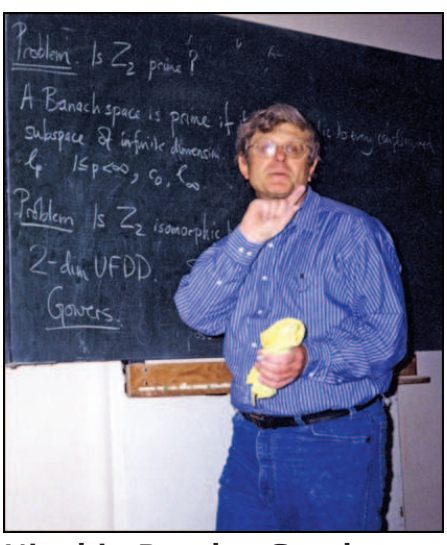
Nigel in Paseky, Czech Republic. luckiest move of my whole professional life: I sent him a preprint on Riesz sets where, among other things, $L^{p}$ spaces with $p$ less than 1 were used. Upon receiving this paper, Nigel discussed it with Elias and Paula Saab, his colleagues from Columbia who knew me from their studies in Paris. A phone call from Elias to my home followed, and I discovered Missouri, where I was to spend, between 1985 and 1997, five of the best years of my life.

These years were illuminated by an exclusive intellectual challenge: collaborating with Nigel. It is hard to explain what this really means. Let me say that working with Nigel was like trying to follow a racing car on a bike. This looks hard, and indeed it is. But what makes it possible and actually rewarding is that the driver understands fully how the biker feels and makes sure to wait for him on every hilltop and to keep waiting until the biker catches his breath before going on. The result is, of course, a rewarding intellectual experience, leading to a wealth of results. This is not a dream. This is much nicer than that.

Nigel Kalton enjoyed all the abilities which are met in first-rate mathematicians: instant understanding of articles or lectures, outstanding computational power, a mathematical instinct for finding the proper original point of view, a baffling memory, and last but not least, a creative mind. He usually did not write much, except for some explicit computations, and I don't think he ever used computers in a proof. The whole work took place

Gilles Godefroy is professor of mathematics at the Institut de Mathématiques de Jussieu. His email address is godefroy@math.jussieu.fr. 


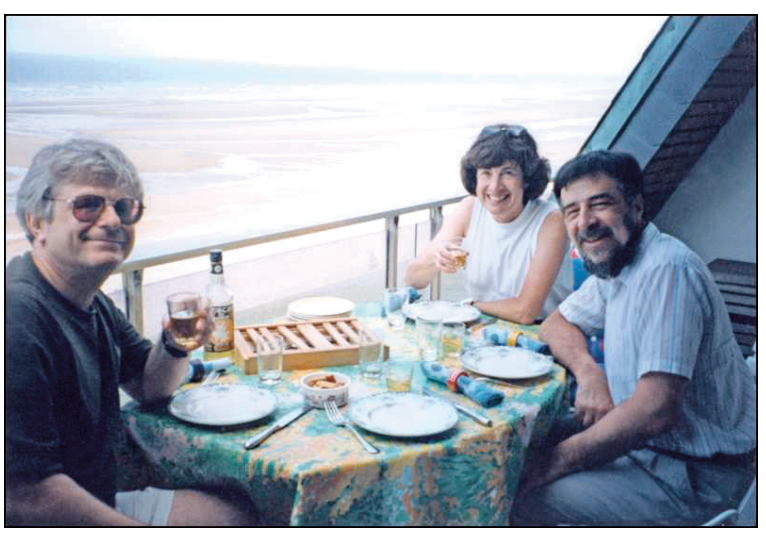

Nigel, Jenny and Gilles Godefroy in Normandy.

in his brain, and I can testify to the fact that he was able to solve highly nontrivial problems while talking about a completely different topic. He was also able to type his articles at high speed and with no scrap paper around him, however complex the arguments were. But on top of all that, Nigel had features which are not always shared by the very few who enjoy similar abilities: although he was entirely self-sufficient, he would listen to all those who approached him with sensible questions, and his attention would soon induce a drastic change in the mathematical landscape! Moreover, Nigel was also working for the future by establishing the proper framework in which a given theory should develop. He was not only solving a problem by going straight to the point but actually analyzing why the problem had been solved, how to attack related ones, and where the power of the existing tools would stop. Such a frame is outlined below.

How did Nigel learn all that he knew? Of course I cannot precisely talk about the early years, but his older brother, Graham, who is a statistician, explained that Nigel discovered alone, at age twelve, a remarkable argument on Poisson distributions. Moreover, his first publication, "Quadratic forms which are perfect squares" (Math. Gazette, 1966), displays work that was completed while Nigel was still in high school. The young Nigel was also an intense chess player, for a while among the twenty best in England. I regret to be unable to say more about this amazing boy who was already standing alone in his class, since Nigel himself did not speak much about his past: it was not in his style to build up a legend and to claim he was a prodigy, although in my opinion this was clearly the case. What I know is that the mature Nigel was seldom reading books or articles. His attitude was rather to rebuild by himself and from scratch what he needed in his work. It was indeed his way of saving time. This did not prevent him, however, from being very careful about credits and references: he knew that there was a theory, developed by such and such, which said that... and thus he would quote the relevant papers. But the theory was in Nigel's mind anyhow. Let me call this a mystery for lack of a better word.

I should now say a few words on Nigel Kalton's mathematics. His articles were dense and deep, and each one of them offered a new point of view. Although Nigel solved scores of problems, I would not call him a problem solver. He would rather catch a question, think about it for a (usually short) while, and then build a device to crunch not only the original problem but actually much more. He frequently stated his theorems in the greatest generality, certainly not for pedantry, but simply because his proofs reached this level. Let me illustrate this point: Nigel was the undisputed world leader on what I call the "Kalton zone", namely, $0 \leq p<1$, where, e.g., the $L^{p}$-spaces cease to be locally convex. His goal was not to focus on marginal objects or pathologies but to explore their deep interplay with Banach spaces and other mainstream objects. An outstanding example of this interplay is provided by Nigel's work on interpolation of Banach spaces, which I now outline.

Nigel Kalton's approach to this theory was to envision the collection of Banach spaces as a manifold, somewhat similar to a Lie group on which smooth lines are defined: the simplest example is provided by the line of $l_{p}$ spaces. Although the points (spaces) on such a complex interpolation line are not isomorphic to each other, they are shown to be close to each other with respect to weak distances, such as the Gromov-Hausdorff distance, which stems from the theory of metric spaces. Moreover, a differentiation functor can be defined and the "tangent vector" at a point $X$ to an interpolation line is a twisted sum of $X$ with itself, which is a quasi-Banach space and sometimes, but not always, a Banach space. Such a twisted sum corresponds to a quasi-linear map $\Omega$ from $X$ to itself, and then any interpolating operator $T$ satisfies commutator estimates, which means that $T$ and $\Omega$ essentially commute. The Hilbert transform is an important example of such an interpolating operator on the $L_{p}$ line.

A fascinating feature of the Kalton calculus is that the analogy with Lie groups can be pursued pretty far, since a "Lie algebra" is available. Indeed, to a sequence space $X$ corresponds a quasi-linear map from the set of positive sequences with finite support to the real line, the entropy function $\Phi_{X}$. This function can be seen as a logarithm of the space $X$, since if, for instance, $X$ is an interpolation space between $X_{0}$ and $X_{1}$, the function 
$\phi_{X}$ is a convex combination of $\Phi_{X_{0}}$ and $\Phi_{X_{1}}$. Conversely, the Kalton calculus provides an exponentiation functor: given a quasi-linear map $\Phi$ satisfying mild necessary conditions, one can construct a space $X$ such that $\Phi=\Phi_{X}$. Since such a $\Phi$ can be written in many ways as a convex combination of quasi-linear maps, a variety of interpolation lines passing through $X$ can be constructed. In other words, extrapolation becomes available-and useful. Indeed, since each interpolation line carries estimates which apply to all interpolating operators, it follows that operators which interpolate around $X$ satisfy a collection of inequalities, yielding to a new symmetric tangent space which is the range of interpolating bilinear forms.

A knight controls the whole game when it reaches a secured square in the middle of a chessboard. Kalton's interpolation theory occupies such a strategic place on the board of functional analysis. Indeed, it provides a unified approach to a score of important objects which were previously unrelated and a canonical way of constructing new ones: quasi-linear maps which are not close to linear ones; twisted Hilbert spaces (as tangent vectors in $l_{2}$ to interpolation lines), among them the Kalton-Peck space which is tangent to the line $\left(l_{1}, l_{\infty}\right)$ and others which are not $U M D$ to well-chosen lines; perturbations of such twisted sums which are explicit examples of complex Banach spaces not complex-isomorphic to their conjugate space; commutator estimates on interpolating operators; nonlinear estimates on $H^{1}(\mathbf{T})$ functions (when the theory is applied to the Hilbert transform on $L^{2}(\mathbf{T})$ ); characterization of trace-class operators on $l_{2}$ which are linear combinations of commutators; twisted sums of spaces with a line which are not locally convex, such as the Ribe space, and so on. This is not the end of the story, but I should not get carried away. Let me simply invite the reader to delve into Nigel's work. One should not be afraid of going deep, all right, but this is a gold mine.

Kalton calculus is one example among many of Nigel's creative power, and I never interfered with his work on interpolation; Michael Cwikel and Stephen Montgomery-Smith would know better. Of course, I could say a few words about my own share among the little crowd of those who were privileged to be Nigel's collaborators: Pete Casazza on bases and approximation theory, Loukas Grafakos on singular integrals, Gilles Lancien on nonlinear geometry of Banach spaces, Jim Roberts on quasi-Banach spaces, Fedor Sukochev on traces and operator ideals, Lutz Weis on $H^{\infty}$ calculus, Dirk Werner on M-ideals...but anyhow, I trust that Nigel's co-authors all share the same memories. Attracting Nigel's attention to a new topic was an exciting and nearly intimidating

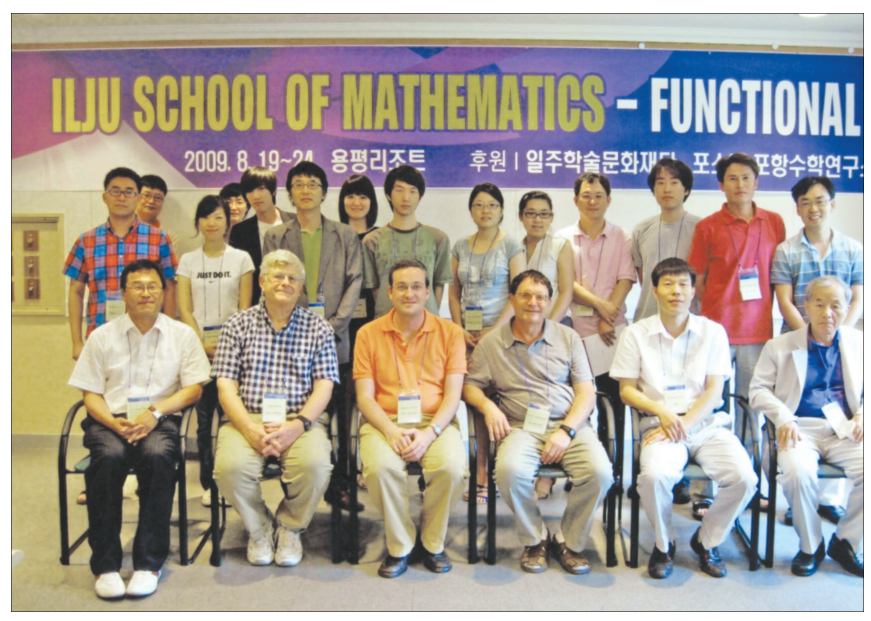

Nigel (front row, second from left) in Korea, 2009.

challenge: big changes were around the corner for sure, but you also knew that in a matter of days he would have gone beyond your most optimistic dreams and you'd have to work hard not to lose sight of the battle scene. There was nothing to be worried about, though; Nigel was a very generous mathematician who would have carried someone on his back rather than leave him/her along the way.

Nigel's mind was constantly in gear, but this did not prevent him from being good company. He was a family man, Jenny's husband for forty-one years and a proud father and grandfather. Sharing time with him was both pleasant and instructive, since besides mathematics he was also a man of culture, with a definite interest in historical matters, and a man of taste who knew how to enjoy good food and good wine-the single topic on which I was, maybe, more knowledgeable than he. There will be more parties, of course, but Nigel cannot be replaced.

His sleepless soul now rests in peace, and we have to keep going. The best we can do is to follow his lead, since Nigel was an achiever who always tried and never gave up. He left us his spirited example and his inspiring mathematics. For all his life he looked ahead, as a giant who sees further than anybody else and shows the way. None of those who approached Nigel Kalton will ever forget him. But as a friend who owes him more than what I can express, I simply wish to say: thank you, Nigel. Once more you are much ahead of me, but kindly waiting as usual. And I hope to meet you again at the top of the ultimate hill. 